ARTICLE OPEN

\title{
Time-restricted feeding induces Lactobacillus- and Akkermansia-specific functional changes in the rat fecal microbiota
}

\author{
Antonio Palomba (iD ${ }^{1,4}$, Alessandro Tanca iD $^{1,2,4}$, Marcello Abbondio iD $^{2}$, Rosangela Sau ${ }^{2}$, Monica Serra ${ }^{3}$, Fabio Marongiu ${ }^{3}$, \\ Cristina Fraumene ${ }^{1}$, Daniela Pagnozzi ${ }^{1}$, Ezio Laconi ${ }^{3}$ and Sergio Uzzau (D) ${ }^{1,2 凶}$
}

Diet is a key factor influencing gut microbiota (GM) composition and functions, which in turn affect host health. Among dietary regimens, time-restricted (TR) feeding has been associated to numerous health benefits. The impact of TR feeding on the GM composition has been mostly explored by means of metagenomic sequencing. To date, however, little is known about the modulation of GM functions by this dietary regimen. Here, we analyzed the effects of TR feeding on GM functions by evaluating protein expression changes in a rat model through a metaproteomic approach. We observed that TR feeding has a relevant impact on GM functions, specifically leading to an increased abundance of several enzymes involved in carbohydrate and protein metabolism and expressed by Lactobacillus spp. and Akkermansia muciniphila. Taken together, these results contribute to deepening our knowledge about the key relationship between diet, GM, and health.

npj Biofilms and Microbiomes (2021)7:85; https://doi.org/10.1038/s41522-021-00256-x

\section{INTRODUCTION}

Lifestyle interventions, including changes in diet and increased exercise, result in many health benefits able to prevent (and enhance treatment of) various metabolic diseases. Among dietary interventions, caloric restriction (CR) has been most intensely investigated. CR consists in a reduction of total daily calories intake without changing the macronutrient composition nor causing malnutrition. Studies in animals and humans have repeatedly reported that $C R$ leads to an extension of both lifespan and healthspan ${ }^{1,2}$. However, the applicability of the $\mathrm{CR}$ regimen is limited, due to the difficulties to be maintained over a long time and to be used in the management of chronic conditions ${ }^{3}$.

An alternative dietary approach, that is being actively explored for its potential benefits, is intermittent fasting (IF). IF regimens were studied both in human subjects and animal models and proved to ameliorate a variety of pathological conditions, including obesity, impaired glucose tolerance, dyslipidemia, hypertension, fertility problems, liver impairment, and neurodegenerative diseases ${ }^{4,5}$. IF has been recently categorized in wholeday fasting, every other day fasting, and time-restricted (TR) feeding ${ }^{6}$. In the TR feeding protocol, food consumption is not randomly distributed across the $24 \mathrm{~h}$, but it is limited to a daily interval of $3-4^{7}, 7-9^{8}$, or $10-12 h^{9}$, allowing daily fasting duration greater than $12 \mathrm{~h}$. A basic rationale of this dietary regimen is that the feeding time period should be aligned with internal circadian rhythms, in order to synchronize with the active phase of animal or human metabolism ${ }^{10,11}$. The benefits of this feeding regimen appear to be proportional to fasting duration ${ }^{6}$. It is important to note that during the TR feeding regimen the quality and quantity of nutrients is comparable to that of ad libitum (AL) controls. In fact, after a short training period (usually lasting less than one week) under controlled experimental conditions, animals exposed to TR feeding are able to eat $\geq 90 \%$ of the food ration consumed by $A L-f e d$ controls $^{12}$. TR feeding, unlike $C R$, can be well tolerated by humans for long periods, also at repeated intervals, as noted in the Islamic fasting during the month of Ramadan ${ }^{13}$. An increasing number of studies have indicated that TR can reproduce at least some of the effects associated with $\mathrm{CR}^{14}$, including stimulation of autophagy, increased mitochondrial respiratory efficiency, modulation of reactive oxygen species, and changes in the profile of inflammatory cytokines ${ }^{15}$. Furthermore, TR was shown to reduce body fat mass ${ }^{16}$, to decrease plasma levels of triglycerides and low-density lipoproteins ${ }^{6,17}$, and to increase lifespan in mice ${ }^{18}$.

Diet is a key factor influencing gut microbiota (GM) composition and functions, which in turn affect host health ${ }^{19}$. It is wellknown that GM composition varies in response to isocaloric diets differing in macronutrient composition, as well as to diets with identical macronutrient composition differing in caloric content ${ }^{20}$. Recently, a few studies specifically investigated the possible impact of TR feeding on GM composition. Ren et al. found alterations in the GM caused by TR feeding linked to recovery from hepatic ischemia-reperfusion injury ${ }^{21}$. Zeb et al. reported that a TR diet can impact the GM, influencing in turn host metabolism and nutritional status ${ }^{22}$. The same research group also showed that TR feeding can enhance GM richness (specifically Prevotellaceae and Bacteroidaceae diversity). Hu et al. demonstrated that a TR feeding regimen in juvenile mice has long-term effects on the GM, leading to a disturbed microbiota-host relationship that can hardly be solved in later life $\mathrm{e}^{23,24}$, suggesting that TR-derived effects might be influenced by the age at which the dietary changes occur.

GM metabolic processes exert a significant impact on the host physiology, affecting gut mucosa homeostasis and being key to the dynamic reciprocal relationship established between the gut and other host systems. In this respect, metaproteomics (unlike metagenomics) can provide reliable information on which biological processes are actually activated (or repressed) by the $\mathrm{GM}$ in response to host or environmental stimuli ${ }^{25}$, by measuring

${ }^{1}$ Porto Conte Ricerche Srl, Science and Technology Park of Sardinia, Alghero, Italy. ${ }^{2}$ Department of Biomedical Sciences, University of Sassari, Sassari, Italy. ${ }^{3}$ Department of Biomedical Sciences, University of Cagliari, Cagliari, Italy. ${ }^{4}$ These authors contributed equally: Antonio Palomba, Alessandro Tanca. ${ }^{凶}$ email: uzzau@uniss.it 
variations occurring in microbial protein abundance and in the corresponding biochemical pathways. Furthermore, metaproteomics can infer which members of the GM are involved in specific molecular functions. However, no studies to date have investigated the influence of TR feeding on GM through a metaproteomic approach.

In the present study, we aimed to investigate the effects of long-term TR feeding on GM protein expression in a rat model, by exploiting an established fecal metaproteomic approach allowing a taxon-specific functional characterization of the GM. In parallel, samples were analyzed by $16 \mathrm{~S}$ rRNA gene sequencing, to provide a gold-standard evaluation of GM taxonomic structure. Taxonspecific processes and pathways possibly influenced by TR feeding were examined and discussed.

\section{RESULTS}

\section{Experimental design and general metrics}

Figure 1 shows the experimental design of the study. After 8 weeks of AL feeding, 16 rats were divided into two groups of 8 rats each: the first group was kept on an AL regimen, whereas the second shifted to TR feeding. Food intake and body weight curves regarding an experiment based on the same dietary regimens and carried out on the same type of rats have been previously published ${ }^{12}$.

After 48 weeks of dietary regimen, stool samples were collected from each rat and divided into two fractions. The first stool fraction was subjected to DNA extraction and 16S rRNA gene amplification and sequencing, for a preliminary investigation of GM taxonomic composition through a widely established approach. The second stool fraction underwent protein extraction and filter-aided sample preparation (FASP) protocol for clean-up, alkylation, and digestion; the so obtained peptide mixtures were then analyzed by liquid chromatography coupled with high-resolution mass spectrometry, according to a shotgun metaproteomics approach, to carry out a functional characterization of the GM.

Concerning 16S rRNA gene sequencing data (for details see Supplementary Data 1), a total of 961,072 reads were obtained from rat fecal samples $(60,067$ on the average per sample), corresponding to 1510 amplicon sequence variants (ASVs). These, in turn, were taxonomically assigned to 51 families, 88 genera, and 43 species (of which 25 with complete binomial nomenclature), as described in Supplementary Data 2.

Concerning metaproteomic data (for details see Supplementary Data 3), we selected four different sequence databases for peptide search (see Methods for further details), namely two collections of rat metagenomic sequences (DB1, containing in-house generated sequences, and DB2, a publicly available database), a rat reference proteome (DB3) and a food database (DB4). A total of 37,341 different peptide sequences were identified and quantified (21,300 on average per sample), distributed as follows among the four databases: $41 \%$ in common between DB1 and DB2, $29.5 \%$ exclusively from DB1, 20.5\% exclusively from DB2, 7.5\% from DB3 and $1.5 \%$ from DB4. Initially, we aimed at identifying the best performing approaches for taxonomic and functional annotation

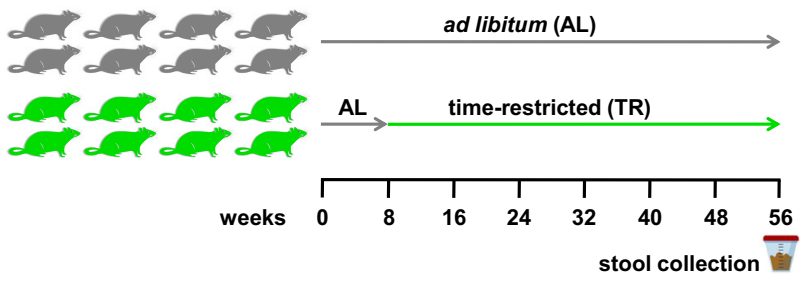

Fig. 1 Experimental design. Schematic representation of the experimental design of the study. of the rat metaproteome, as shown in Supplementary Data 4. First, we compared the performance of Unipept (tryptic peptide-, UniProt-based tool) and MEGAN (protein BLAST-, NCBI-based tool) regarding taxonomic annotation. Annotation yields reached by MEGAN were almost double than those reached by Unipept at all levels (e.g., $28.4 \%$ vs. $12.8 \%$ of peptide sequences annotated at the genus level), although Unipept led to much higher richness levels (e.g., 312 vs. 39 different taxonomic genera detected in the study). Based on the better annotation yield, we chose MEGAN as a taxonomic annotation tool for this study. According to the MEGAN taxonomic classification, 30,331 peptide sequences $(17,331$ on the average per sample) were assigned to microbial taxa, while 2709 peptide sequences (1695 on the average per sample, corresponding to 527 proteins) were assigned to the host (rat).

Then, we compared the performance of two functional annotation approaches: (i) alignment of protein sequences against a Swiss-Prot database and retrieval of "protein family" information from UniProt, and (ii) processing of protein sequences with the eggNOG-mapper tool and retrieval of "KEGG Orthology Groups" (KOGs). The latter approach provided the best performance, with $69 \%$ of peptide sequences annotated vs. $57 \%$ with the former approach. Therefore, KOGs were chosen as the main category for functional annotation, with $1387 \mathrm{KOGs}$ detected in total in the study. Other levels of functional annotation, such as the more generic "Cluster of Orthologous Groups" (COGs) and the more specific "Carbohydrate-Active enzymes" (CAZy) and "metabolic pathways", were also used in various points of this study.

Statistics of combined taxonomic-functional annotations are provided in Supplementary Data 5.

\section{Taxonomic changes induced by TR feeding in the rat fecal microbiota based on 16S rRNA gene sequencing}

To verify whether TR diet could affect the structure of the rat GM, we investigated its taxonomic composition through 16S rRNA gene sequencing. After a preliminary unsupervised data evaluation through Principal Coordinate Analysis (PCoA; Supplementary Fig. 1), we compared the GM structures of TR- and AL-fed rats with the aim of identifying differentially abundant families (Supplementary Fig. 2), genera (Fig. 2) and species (Supplementary Fig. 3). We found the lineage Akkermansiaceae/Akkermansia/A. muciniphila, as well as Prevotellaceae (among families), Anaerovorax and Marvinbryantia (among genera) and Ruminococcus flavefaciens (among species, although the corresponding genus went in the opposite direction, as stated below), among taxa enriched in the GM of TR-fed compared to AL-fed rats. On the other hand, several taxa resulted higher in AL-fed compared to TR-fed rats, such as

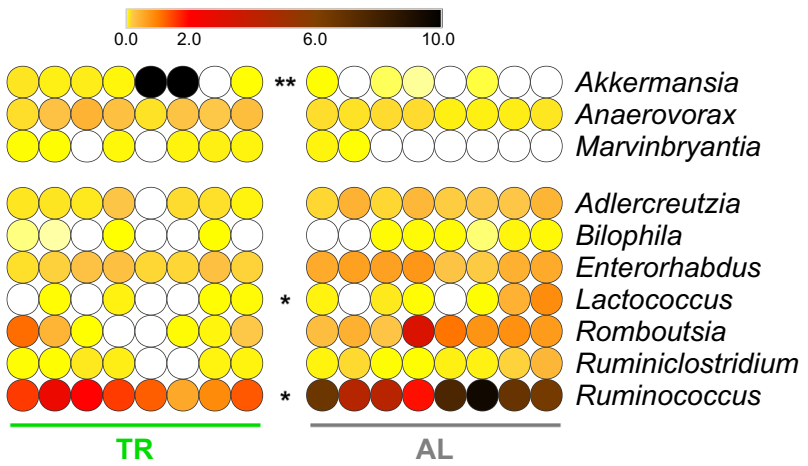

Fig. 2 Changes in taxonomic genus composition in the fecal microbiota of TR- vs. AL-fed rats according to 16S rRNA gene sequencing results. Heatmap illustrating genera with significantly differential abundance between $A L$ and $T R$ groups ( $F D R<0.1$ ). A single or double asterisk refers to $\mathrm{FDR}<0.01$ or $<0.001$, respectively. Each dot indicates a different sample. The color gradient is based on the relative abundance of the genus. 
Atopobiaceae, Erysipelotrichaceae and Ruminococcaceae among families, Adlercreutzia, Enterorhabdus, Bilophila, Lactococcus, Romboutsia, Ruminiclostridium, and Ruminococcus among genera, as well as two species belonging to the genus Bacteroides (namely, $B$. rodentium and $B$. thetaiotaomicron). Complete results of differential analysis based on 16S rRNA gene sequencing data are shown in Supplementary Data 6.

\section{Taxonomic changes induced by TR feeding in the rat fecal microbiota based on metaproteomic data}

The fecal samples analyzed by $16 \mathrm{~S}$ rRNA gene sequencing were further investigated to characterize the rat GM metaproteome. Initially, we focused on the taxonomic data, performing a

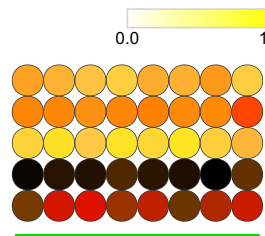

TR

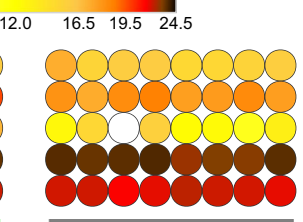

AL
Acetatifactor Akkermansia Faecalibaculum Lactobacillus Oscillibacter

Fig. 3 Changes in taxonomic genus composition in the fecal metaproteome of TR- vs. AL-fed rats. Heatmap illustrating microbial genera with significantly differential abundance between $A L$ and TR groups (FDR $<0.1$ ). Each dot indicates a different sample. The color gradient is based on the relative abundance of the genus. preliminary unsupervised data evaluation via PCoA (Supplementary Fig. 4) and then a differential analysis between the two experimental groups at the family (Supplementary Fig. 5), genus (Fig. 3), and species (Supplementary Fig. 6) level. Among taxa enriched in the GM of TR-fed compared to AL-fed rats, we found again Akkermansia/A. muciniphila, together with Lactobacillaceae/ Lactobacillus/L. reuteri, Oscillospiraceae/Oscillibacter, Desulfovibrionaceae, Acetatifactor/A. muris, Faecalibaculum/F. rodentium and Treponema succinifaciens. The only taxon being more abundant in AL-fed compared to TR-fed rats was Eubacterium plexicaudatum. Complete results of differential analysis based on metaproteomic taxonomic data are shown in Supplementary Data 7.

\section{Taxon-specific functional changes induced by TR feeding in the rat fecal microbiota}

Next, we focused on the key output of metaproteomics, i.e., the functional analysis of the GM. Specifically, we combined functional (KOG) and taxonomic (family, genus, or species) information assigned to each peptide sequence. After the PCoA unsupervised evaluation (Supplementary Fig. 7), a differential analysis between the two experimental groups was performed, which led to the identification of numerous taxon-specific differential functions, listed in Supplementary Fig. 8 (family-specific functions), Fig. 4 (genus-specific functions), and Supplementary Fig. 9 (speciesspecific functions). As shown in the Figures, most of the differential functions were expressed by mucus colonizers Lactobacillus and Akkermansia members.

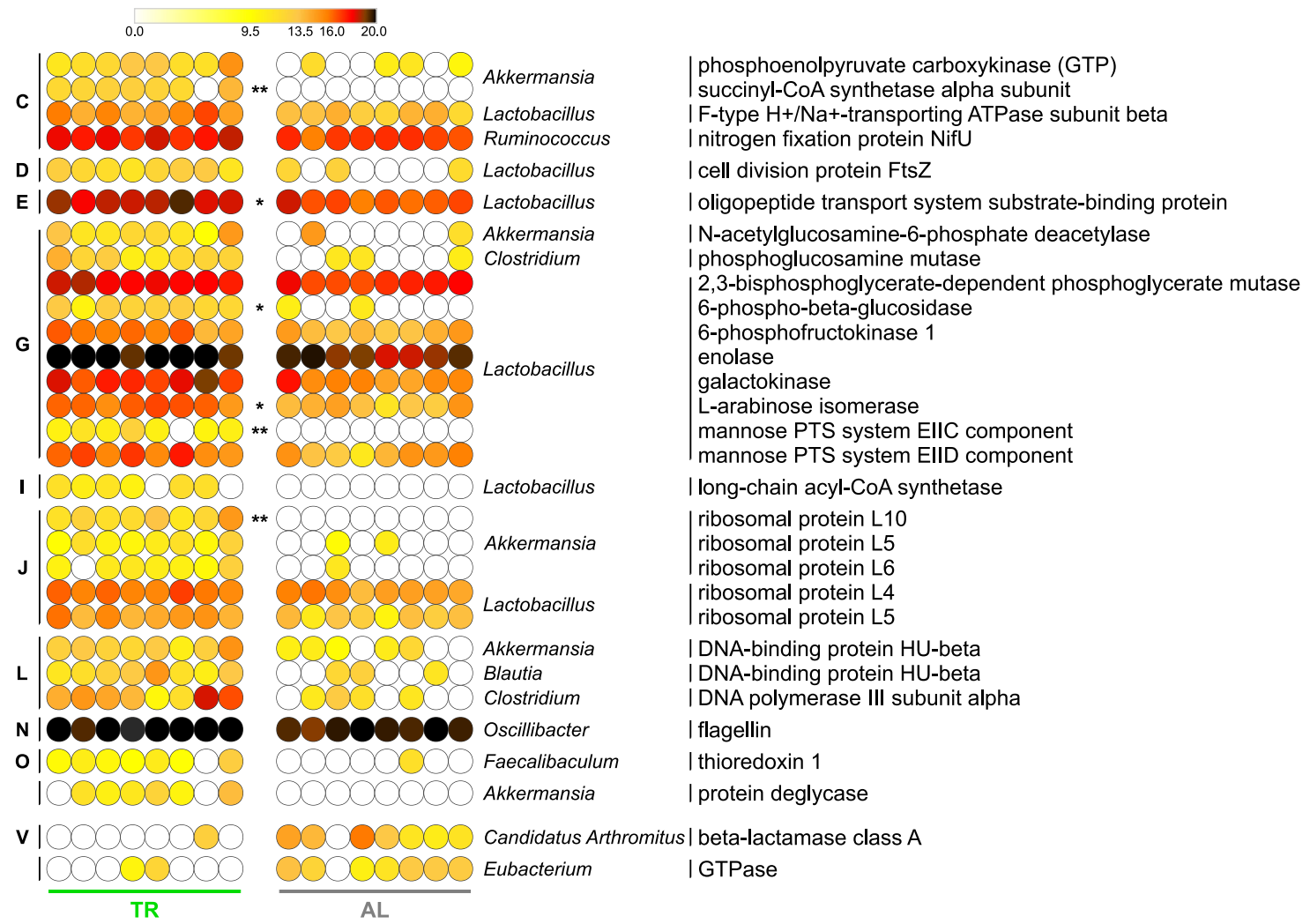

Fig. 4 Changes in the functional profile of the fecal metaproteome of TR- vs. AL-fed rats. Heatmap illustrating genus-specific functions with significantly differential abundance between $A L$ and TR groups (FDR $<0.1$ ). A single or double asterisk refers to FDR $<0.01$ or $<0.001$, respectively. Each dot indicates a different sample; the color gradient is based on the relative abundance of functions. Functions are ordered sequentially: (i) according to the group of rats in which they are significantly more abundant, indicated in the bottom; (ii) according to their COG category, indicated close to the left margin of the heatmap (C, Energy production and conversion; $D$, Cell cycle control, cell division, chromosome partitioning; E, Amino acid transport and metabolism; G, Carbohydrate transport and metabolism; I, Lipid transport and metabolism; J, Translation, ribosomal structure and biogenesis; L, Replication, recombination and repair; N, Cell motility; O, Posttranslational modification, protein turnover, chaperones; V, Defense mechanisms); (iii) according to the taxonomic genus to which they were assigned, indicated close to the right margin of the heatmap; (iv) in alphabetical order. 
Specifically, numerous Lactobacillus/Lactobacillaceae enzymes were detected as significantly more abundant in the GM of TRfed rats, involved in glycolysis (phosphoglycerate mutase, enolase, 6-phosphofructokinase), galactose metabolism (galactokinase, alpha-galactosidase), pentose and glucuronate interconversions (arabinose isomerase), fatty acid oxidation (longchain acyl-CoA synthetase) and glycan degradation (6-phosphobeta-glucosidase, belonging to the glycosyltransferase family GT1). Moreover, the list of microbial functions induced by TR feeding included proteins involved in cell division (cell division protein FtsZ), carbohydrate transport (mannose PTS system components), and peptide transport (oligopeptide transport system substrate-binding protein).

Furthermore, the following protein functions are expressed by Akkermansiaceae/Akkermansia/A. muciniphila were found higher in TR-fed rats: several enzymes, including succinyl-CoA synthetase (TCA cycle), phosphoenolpyruvate carboxykinase (gluconeogenesis), N-acetylglucosamine-6-phosphate deacetylase (aminosugars metabolism/peptidoglycan recycling), and protein deglycase (protein/nucleotide repair), as well as histone-like (DNA-binding protein HU-beta) and ribosomal proteins.

Interestingly, the only two functions higher in AL-fed rats were a beta-lactamase class A from Candidatus Arthromitus and a GTPase from Eubacterium. Noteworthy, no significant differences in abundance could be found concerning host (rat) protein functions.

Aggregating data at a more general annotation level, namely "metabolic pathway", we found that several Lactobacillus-specific pathways, including L-arabinose degradation via L-ribulose, galactose metabolism and peptidoglycan biosynthesis, resulted as significantly more abundant in TR-fed rats.

Complete results of differential analysis based on metaproteomic functional and combined taxonomic-functional data are shown in Supplementary Data 8 and 9, respectively.
Proteomic profiles of the main fecal microbiota members in rats subjected to TR feeding

To gain insight into the contribution of specific members to the functional activity of the GM in TR- and AL-fed rats, we investigated the proteome profile expressed by the two most represented Lactobacillus species, namely $L$. murinus and $L$. reuteri. As shown in Fig. 5, the two species-specific functional patterns differed considerably, with filamentous hemagglutinin being the only common protein, L. murinus being more involved in carbohydrate transport and metabolism, and L. reuteri mostly exerting functions related to amino acid and nucleotide metabolism. To have a broader picture, the complete Lactobacillus proteome (genus-specific functions detected in all the samples) is presented in Supplementary Fig. 10, with differentially abundant functions in bold (in common with Fig. 4).

Furthermore, Akkermansia-specific functions are listed in Supplementary Fig. 11. Of note is the presence of enzymes involved in aminosugar metabolism, citric acid cycle, and response to oxidative stress.

\section{TR feeding promotes the expression of galactose metabolism enzymes in Lactobacillus}

Examining enzymatic functions showing abundance changes associated with TR feeding and assigned to lactobacilli, we noticed that a group of them belong to the galactose metabolism pathway. Therefore, we decided to inspect the abundance data of all enzymes potentially involved in the degradation and biosynthesis of galactose-containing glycans, with a special focus on the Leloir pathway (as shown in Fig. 6).

Interestingly, all enzymes converging on this pathway, identified in this study and attributable to lactobacilli (taxonomic levels from order Lactobacillales down to genus Lactobacillus are
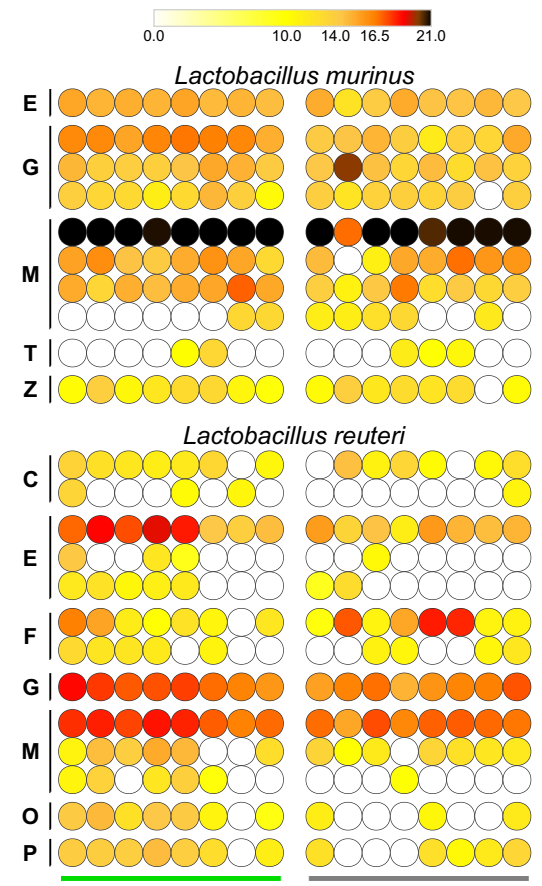

TR

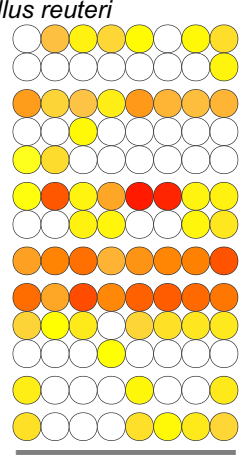

$\mathrm{AL}$ glycine hydroxymethyltransferase

L-arabinose isomerase

multiple sugar transport system ATP-binding protein

sucrose phosphorylase

filamentous hemagglutinin

Ca-activated chloride channel homolog

large repetitive protein

IgA-specific serine endopeptidase

two-component system, chemotaxis family, sensor kinase CheA

rod shape-determining protein $\mathrm{MreC}$

pyruvate dehydrogenase E2 component (dihydrolipoamide acetyltransferase)

pyruvate dehydrogenase $\mathrm{E} 1$ component alpha subunit

urease subunit beta

arginine deiminase

glutamine synthetase

deoxyribose-phosphate aldolase

purine nucleosidase

xylulose-5-phosphate/fructose-6-phosphate phosphoketolase

filamentous hemagglutinin

general secretion pathway protein $B$

choloylglycine hydrolase

HSP20 family protein

starvation-inducible DNA-binding protein

Fig. 5 Lactobacillus murinus/reuteri-specific functions detected in fecal metaproteome samples of TR- and AL-fed rats. Functions in bold are differentially abundant between the two groups (FDR $<0.1$; see Supplementary Fig. 9). Each dot indicates a different sample. The color gradient is based on the relative abundance of functions. Functions are ordered sequentially: (i) according to their COG category, indicated close to the left margin of the heatmap (C, Energy production and conversion; $E$, Amino acid transport and metabolism; $F$, Nucleotide transport and metabolism; G, Carbohydrate transport and metabolism; M, Cell wall/membrane/envelope biogenesis; $\mathrm{O}$, Posttranslational modification, protein turnover, chaperones; P, Inorganic ion transport and metabolism; T, Signal transduction mechanisms; Z, Cytoskeleton); (ii) according to their average relative abundance. 


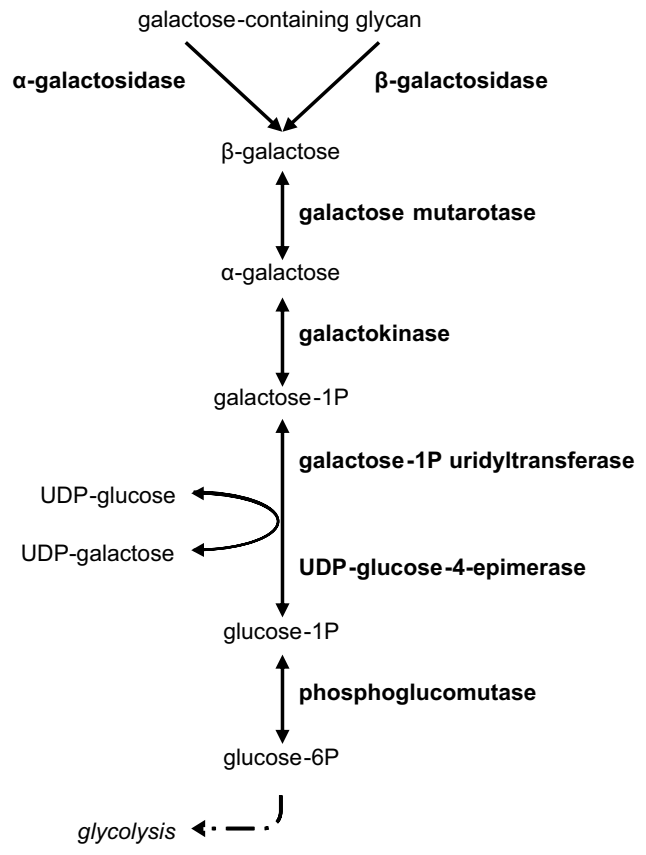

\begin{tabular}{|c|c|c|c|c|}
\hline enzyme & taxon & $p$-value & FDR & $L R(T R / A L)$ \\
\hline \multirow{4}{*}{$\alpha$-galactosidase } & all & 0.01522 & 0.29538 & 1.04 \\
\hline & Lactobacillales & 0.00349 & 0.07723 & 2.81 \\
\hline & Lactobacillaceae & 0.00546 & 0.09753 & 2.81 \\
\hline & Lactobacillus & 0.01110 & 0.14832 & 2.81 \\
\hline \multirow{4}{*}{$\beta$-galactosidase } & all & 0.34329 & 0.76343 & 0.36 \\
\hline & Lactobacillales & 0.04555 & 0.31693 & 1.31 \\
\hline & Lactobacillaceae & ND & ND & ND \\
\hline & Lactobacillus & ND & ND & ND \\
\hline \multirow{4}{*}{ galactose mutarotase } & all & 0.38952 & 0.78406 & 0.10 \\
\hline & Lactobacillales & ND & ND & ND \\
\hline & Lactobacillaceae & ND & ND & ND \\
\hline & Lactobacillus & ND & ND & ND \\
\hline \multirow{4}{*}{ galactokinase } & all & 0.00201 & 0.07745 & 1.28 \\
\hline & Lactobacillales & 0.00086 & 0.03323 & 1.80 \\
\hline & Lactobacillaceae & 0.00086 & 0.03354 & 1.80 \\
\hline & Lactobacillus & 0.00086 & 0.03240 & 1.80 \\
\hline \multirow{4}{*}{ galactose $-1 P$ uridyltransferase } & all & 0.16223 & 0.60371 & 0.05 \\
\hline & Lactobacillales & ND & ND & ND \\
\hline & Lactobacillaceae & ND & ND & ND \\
\hline & Lactobacillus & ND & ND & ND \\
\hline \multirow{4}{*}{ UDP-glucose-4-epimerase } & all & 0.24296 & 0.68189 & 0.35 \\
\hline & Lactobacillales & 0.00859 & 0.11940 & 2.38 \\
\hline & Lactobacillaceae & 0.01185 & 0.16825 & 2.38 \\
\hline & Lactobacillus & 0.01919 & 0.21072 & 2.38 \\
\hline \multirow{4}{*}{ phosphoglucomutase } & all & 0.28648 & 0.72744 & 0.25 \\
\hline & Lactobacillales & 0.01938 & 0.19946 & 2.26 \\
\hline & Lactobacillaceae & 0.02097 & 0.22777 & 2.26 \\
\hline & Lactobacillus & 0.02410 & 0.24435 & 2.26 \\
\hline
\end{tabular}

Fig. 6 Leloir pathway, galactose metabolism, and lactobacilli in the fecal microbiota of TR- vs. AL-fed rats. Left, schematic representation galactose metabolism enzymes (bold) and metabolites, with focus on the Leloir pathway. Right, taxonomic annotation of Leloir pathway/ galactose metabolism enzymes detected in the samples analyzed in this study. $p$-value, FDR, and log-ratio (LR) value obtained upon TR vs. AL differential analysis are reported for each enzyme-taxon combination. $p$-values $<0.05$, FDRs $<0.1$ and LRs $>1$ are shown in red; FDR values $<$ 0.1 are also in bold. ND, not detected (or not passing the filters).

presented), exhibited a higher mean abundance in the metaproteome of TR-fed rats together with a non-adjusted $p$-value $<$ 0.05 , even if the false-discovery rate (FDR) threshold (0.1) was passed in only two cases. This suggests the existence of a differential galactose metabolism-related trend induced by TR feeding.

\section{DISCUSSION}

Diet is known as one of the most important factors influencing GM. Recently, some research groups have explored the impact of TR feeding on GM structure, but no information is available about its effect on GM functions. Fasting, per se, is associated with increased gastrointestinal transit ${ }^{26}$. When fasting is extended for up to $12 \mathrm{~h}$, gastrointestinal transit of ingested food is expected to be completed before the intake of new food ${ }^{27}$. Hence, the small intestine, cecum, and proximal colon are expected to be relatively free of dietary nutrients for about $12 \mathrm{~h}$ a day. Endogenous substrates available for microbes colonizing the distal ileum, cecum, and colon are mainly derived from host secreted proteins and peptides, exfoliated cells, and mucins (of salivary, gastric, bronchial, hepato-biliary, small intestinal, and colonic origin). While secretions from the pancreas and other glands are stimulated by the entry of the acidic chyme into the duodenum, mucins are produced continuously and represent a continuous source of proteins and carbohydrates for the "fasting" GM. In this scenario, although challenging, metaproteomic analyses have the potential for a more complete understanding of the GM ecosystem than sequencing alone. Metaproteome data include both taxonomic and functional annotations of proteins, enabling the characterization of the main metabolic pathways activated in the most abundant GM members. Here, using fecal samples obtained from a rat model, we investigated the variation of microbial protein expression in rats subjected to long-term TR feeding. On the other hand, the choice of the fecal sample might be considered as a limit of this study, since fecal microbiota strongly resembles large intestine microbiota, whereas it differs from small intestine microbiota ${ }^{28}$. This sample should be therefore regarded as a reliable proxy for investigating colonic microbial communities; however, considerably different data might be obtained as far as small intestine samples are analyzed.

Differential composition of the GM in TR- vs. AL-fed rats was initially assessed in this study by $16 \mathrm{~S}$ rRNA gene sequencing. This approach highlighted significant changes in several taxa, including bacteria that are well-known members of the mucosaassociated microbiota (MAM): Akkermansia, Bacteroides (i.e., B. rodentium and $B$. thetaiotamicron), Bilophila, Marvinbryantia, and Ruminiclostridium $^{29,30}$. Two species, A. muciniphila and B. rodentium, showed the highest relative abundance fold changes (in absolute terms) when comparing TR- and AL-fed rats. Noteworthy, the increased abundance of $A$. muciniphila in TR-fed rats $\left(\log _{2} \mathrm{FC}=\right.$ 7.3; $F D R=0.0001$ ) is consistent with its mucin-degrading capacity, outperforming other members of the MAM in growth and replication during fasting periods, when mucins are the sole source of nutrients ${ }^{31}$; on the other hand, $B$. rodentium, as well as $B$. thetaiotamicron, is a glycan generalist and mucus degrader and its growth and replication might be fueled more efficiently when a more complex and abundant assortment of diet-derived glycans are available at the mucosa surface for longer periods, as expected in $\mathrm{AL}$-fed rats $\left(\log _{2} \mathrm{FC}=-5.46 ; \mathrm{FDR}<0.000001\right)^{32,33}$.

A. muciniphila increased abundance in TR-fed rats is of particular interest. This species is considered a keystone member of the MAM, affecting metabolisms of other commensals ${ }^{34}$. Further, $A$. muciniphila is one of the most relevant species reported to be involved in the complex relationships between diet and metabolic/inflammatory diseases ${ }^{35-38}$. Long-term, high-calorie diets have been already demonstrated to promote the reduction of Akkermansia relative abundance and the increase of pathobiont Bilophila $^{39}$. An opposite trend by Akkermansia and Bilophila was observed also in our TR-feeding rat model, suggesting that, while 
TR feeding might protect against metabolic diseases ${ }^{6,8,11,16}$, its impact on the GM composition might recapitulate that obtained with a balanced host metabolic state.

Our metaproteomic analysis showed that TR feeding is able to induce changes in GM metabolic pathways. Most of the differential functions identified in this study were expressed by members of the mucus colonizers Akkermansia and Lactobacillus. TR-feeding increased the relative abundance of a specific set of Akkermansia-specific proteins, including several metabolic enzymes, such as succinyl-CoA synthetase, phosphoenolpyruvate carboxykinase, N-acetylglucosamine-6-phosphate deacetylase, and protein deglycase. Although no enzymes directly and explicitly involved in mucin degradation could be detected (possibly due to an abundance level lower than the detection limit of the analytic pipeline used), N-acetylglucosamine-6phosphate deacetylase is known to be implicated in reactions linking mucin degradation to glycolytic/gluconeogenetic pathways, as well as to peptidoglycan biosynthesis ${ }^{40}$. In addition, some of the differential enzymatic functions were not even detectable in the GM of AL-fed rats, suggesting that they might be tightly regulated when the influx of dietary compounds occurs in continuous. Strikingly, the differential abundance of the DNAbinding protein HU-beta might be part of this control in TR-fed rats, given its role in active DNA metabolic transactions ${ }^{41,42}$. This protein stabilizes DNA conformations required to promote and sustain the regulation of many enzymes involved in energy metabolism and catabolism pathways ${ }^{43}$. We hypothesize that the growth of $A$. muciniphila in TR-fed rats may be sustained prevalently by mucin, leading to a blooming of this species. A. muciniphila might exploit the advantage of continuous availability of mucin as a nutritional source by a tight (HU protein-dependent) control over the expression of metabolic unnecessary functions, in response to the energy requirement in the fasting gut environment. Furthermore, according to the recently reported ecological co-exclusion behavior of the Akkermansia species $^{44}$, we believe that the TR feeding-dependent increase of Akkermansia genomic copies and protein functions, observed side by side in the present study, are referred uniquely to strains belonging to A. muciniphila.

A further interesting finding of our metaproteomic analyses was the increased abundance of functions expressed by L. reuteri and, overall, by the Lactobacillus/Lactobacillaceae taxa in the fecal microbiota of TR-fed rats. However, in this case, sequencing analyses showed no differential distribution of Lactobacillus species between the two groups of rats. The lack of consensus between 16S rRNA gene sequencing and metaproteomics GM data has already been widely acknowledged as probably due to the different methods of molecular target extraction, differences in the sequences database chosen, and amplification/identification biases ${ }^{45-47}$. Nonetheless, previous DNA sequencing studies reported an increase in the relative proportion of Lactobacillus spp. in the GM of rodents subjected to CR or $\mathrm{IF}^{26-30}$. Probably, the 16S rRNA gene sequencing pipeline used in this study was less sensitive than the metaproteomic pipeline and did not allow us to confirm previous reports. Lactobacilli are the most numerous and diverse group among lactic acid bacteria that inhabit mucosal surfaces of the oral cavity and the gastrointestinal tract in many animal species, including humans and rodents ${ }^{27,48}$. Lactobacilli are also known to adhere to the intestinal surface through the interaction with mucins, as well as to be able to induce mucin production ${ }^{49}$. In addition, lactobacilli possess inducible proteolytic activity enabling digestion of mucin backbones, and their relative abundance was found to be significantly decreased in $M u c 2^{-1-}$ mice ${ }^{50}$. Consistently, sequencing of numerous Lactobacillus genomes has revealed a wide assortment of genes encoding glycoside hydrolases active on carbohydrate moieties of host mucins and oligosaccharides ${ }^{51}$. Of note, the presence of mucindegrading bacteria in the GM, when combined with a fiber-rich diet, has been associated to an increased gut health ${ }^{52-54}$. This might lead to hypothesize a link between TR feeding, mucin degradation, and intestinal homeostasis. In addition to the $A$. muciniphila-specific differential functions described above, we also observed that several Lactobacillus-specific enzymes are involved in the metabolism of galactose (likely, in the degradation of galactose-containing glycans) and taxonomically assigned to lactobacilli were clearly more abundant in the animals subjected to TR diet. Since TR- and AL-fed rats were administered the same (low-fat) diet, we can speculate that the higher expression by lactobacilli of enzymes involved in galactose metabolism in the GM of fasted rats might be induced by a relatively increased access to the main endogenous source of galactose, namely, gut mucins. Furthermore, monosaccharide and oligopeptide transporters were found as significantly more expressed by Lactobacillus spp. colonizing TR-fed rats, possibly related to the uptake of carbohydrates and peptides originated from catabolism. Other differential Lactobacillus-specific functions identified in this study can shed light on further biological mechanisms associated to TR feeding. Cell division protein FtsZ resulted among the Lactobacillus-specific functions more abundant in the GM of TR-fed rats compared to AL-fed rats. Given its rapid degradation, the dynamics of FtsZ concentration have been elegantly shown to predict cell division rate, particularly in environments where nondividing microbes receive nutrients in small quantities, such as in the gut ${ }^{55}$. Therefore, the higher abundance of cell division protein FtsZ might account for an increased rate of Lactobacillus replication in TR-fed rats. Another of the enzymes varying their abundance in TR-fed animals was arabinose isomerase, which catalyzes the conversion of L-arabinose (usually produced by other microbes able to degrade plant polysaccharides) to L-ribulose, confirming the existence of cross-feeding mechanisms. Interestingly, previous studies reported the ability of some Lactobacillus strains to secrete this enzyme, causing an anti-hyperglycemic effect in mice ${ }^{56}$.

Besides Akkermansia and Lactobacillus, this study reports other (less abundant and less known) GM members as possibly influenced by TR feeding. Namely, Faecalibaculum abundance was significantly increased in TR-fed rats according to metaproteomic data. Previously, in a mouse model of Alzheimer's disease, Faecalibaculum was found as more abundant in CR-fed animals compared to AL-fed ones; moreover, in the same animals, both Faecalibaculum and Lactobacillus have been strongly associated with a protective effect, i.e., a reduction of amyloid-beta plaque deposition ${ }^{57}$. As for Lactobacillus and A. muciniphila, TR-dependent changes in the gut mucosal ecosystem (i.e., the extended availability of host mucus as the main source of nutrients) might be one of the main causes of the relatively increased abundance of Faecalibaculum. In a recent study, Faecalibaculum rodentium has been found to be strongly depleted during tumorigenesis and to be able to reduce tumor growth through the production of shortchain fatty acids ${ }^{58}$; intriguingly, its depletion occurs together with mucus changes. Expansion of Faecalibaculum in TR-treated rats might thus occur as this genus belongs to GM members whose survival and growth appear to be warranted by availability of host mucus, rather than of substrates from the diet. Prevotellaceae were also found to increase in the TR-fed group of rats according to $16 \mathrm{~S}$ rRNA gene sequencing results. This finding is consistent with the recent observation of Wang et al. in swine, where TR feeding-dependent increase of Prevotellaceae abundance was negatively correlated to 2-amino-butyrate, a metabolite previously associated with high cardiovascular risk ${ }^{59}$. Finally, beta-lactamase encoded by Candidatus Arthromitus was detected in higher abundance in AL-fed than in TR-fed rats. While antibioticresistant genes, as part of the GM resistome, have been detected in subjects with no history of antibiotic treatment, their abundance is reported to increase in individuals (obese and overweight) with increased calorie intake ${ }^{60,61}$. 
In conclusion, we observed that TR feeding has a relevant impact on GM functions, according to our rat model. This fastingassociated dietary regimen appears to specifically boost nextgeneration probiotic $A$. muciniphila and several biological activities exerted by other GM members (in particular, proteolytic and galactose metabolism enzymes expressed by Lactobacillus spp.). As mucin-degrading activity has been often related to gut homeostasis, a mechanism linking TR feeding, an increase of beneficial A. muciniphila and Lactobacillus protein functions, degradation of mucin, and intestinal health can be hypothesized. Further investigations integrating DNA sequencing, metaproteomics, and metabolomic analyses, as well as mechanistic and functional studies, are needed to confirm this hypothesis and to deepen our knowledge about molecular aspects of diet-induced changes in the host-microbiota relationship.

\section{METHODS}

\section{Animal intervention and sampling}

This study has been performed with a colony of DPP-IV_Fischer 344 male rats bred in-house at the Department of Biomedical Sciences, University of Cagliari. Rats were maintained on an alternating 12-h light/dark cycle (light on at 7 p.m., light off at 7 a.m.), in a temperature- and humidity-controlled environment, with water available $A L$, and housed two for each cage. Animals were fed AL until the age of 8 weeks with Purina Rodent Lab Chow 4RF21 diet (percentage composition: water $12 \%$, protein $18.5 \%$, fat $3 \%$, fiber $6 \%$, ash 7\%, nitrogen-free extract 53.5\%; Mucedola srl, Settimo Milanese, Italy). After 8 weeks, rats were divided into 2 groups (each of 8 animals, with 2 rats per cage): the AL control group and the TR feeding group. More specifically, the AL group had AL access to food during both light and dark phases, while the TR group had AL access to food for $8 \mathrm{~h}$ during the dark phase, namely from 11 a.m. to 7 p.m. ( $4 \mathrm{~h}$ after lights off). Fecal samples were collected after 48 weeks of diet regimen. Rats were individually placed in a separate cage for fecal sample collection and feces were immediately harvested and stored at $-80^{\circ} \mathrm{C}$. Rats received humane care according to the criteria outlined in the National Institutes of Health Publication 86-23, revised 1985. Animal studies were reviewed and approved by the Institutional Animal Care and Use Committee of the University of Cagliari and were performed in accordance with the relevant ethical guidelines and regulations (authorization of the Italian Health Ministry No. 840/2016-PR).

Fecal samples $(N=16)$ were immediately stored at $-80^{\circ} \mathrm{C}$ until use. At the time of the analyses, stool samples were thawed at $4{ }^{\circ} \mathrm{C}$ and divided into two portions, for DNA and protein extraction, respectively.

\section{DNA extraction and 16S rRNA gene sequencing}

Sixteen fecal samples, collected from rats belonging to AL and TR groups, were subjected to DNA extraction with the QIAamp Fast DNA Stool Mini Kit (Qiagen, Hilden, Germany). The extracted DNA was purified according to E.Z.N.A. Soil DNA Kit (Omega Bio-Tek, Norcross, GA, USA). DNA quality and yield were evaluated via agarose gel and Qubit ${ }^{\mathrm{TM}}$ Fluorometer (Life Technologies, Carlsbad, CA, USA, now Thermo Fisher Scientific). Libraries were constructed using Illumina's recommendations as implemented in the $16 \mathrm{~S}$ Metagenomic Sequencing Library Preparation guide.

The variable region 4 (V4) of the gene encoding the 16S rRNA was amplified using the $515 \mathrm{~F}$ and $806 \mathrm{R}$ primers (GTGCCAGCMGCCGCGGTAA and GGACTACHVGGGTWTCTAAT, respectively), modified to contain adaptors for MiSeq sequencing (Illumina, San Diego, CA, USA). Two separate gene amplification reactions were performed for each sample, and the products were pooled together and cleaned up using Agencourt AMPure XP Beads (Beckman Coulter Genomics, MA, USA). The next PCR attached dual index barcodes using the Illumina Nextera XT kit so that the PCR products may be pooled and sequenced directly. The final quality control and quantification were conducted using the BioAnalyzer 2100 instrument (Agilent Technologies, Santa Clara, CA, USA). Libraries (average size $440 \mathrm{bps}$ ) were quantified with the Qubit ${ }^{\mathrm{TM}}$ Fluorometer, normalized, and then pooled equimolar, including $10 \%$ PhiX as an internal control. DNA sequencing was performed on the Illumina MiSeq platform, using v3 chemistry (following the manufacturer's specifications), to generate pairedend reads of 201 bases in length in each direction.

\section{Analysis of 16S rRNA gene sequencing data}

Primers spanning the $\mathrm{V} 4$ region $(515 \mathrm{~F}$ and $806 \mathrm{R}$ ) were removed from the generated fastq files by cutadapt ${ }^{62}$. Reads were further analyzed with the Quantitative Insights Into Microbial Ecology 2 (QIIME2) pipeline (v.2$2021.2)^{63}$. Initially, DADA2 ${ }^{64}$ was used to inspect reads quality and create sequencing error profiles, truncate (both forward and reverse reads to $180 \mathrm{bp}$ ), assemble read pairs, remove chimeras, and infer the ASVs present. Taxonomy was assigned using pre-formatted SILVA 138 SSURef NR99 ${ }^{65}$ full-length reference sequence and taxonomy files, processed using the RESCRIPt plugin and q2-feature-classifier ${ }^{66}$, provided in the QIIME2 data resources (https://docs.qiime2.org/2021.2/data-resources). A supervised classification method was applied to classify the representative sequences from our V4 dataset, training the Naïve Bayes classifier ${ }^{67}$ using SILVA 138 reference sequences.

Pre-processing of the ASV table was performed using phyloseq ${ }^{68}$ package (v.1.28.0) in R (v3.6.3; https://www.R-project.org). Filtering was done by removing ASVs classified as chloroplast, mitochondria, or without kingdom-level classification from sequencing data. In addition, taxa with unconventional nomenclature were manually filtered out for the differential analysis. The taxa for which a binomial nomenclature was not available are marked in red in Supplementary Data 1.

\section{Protein extraction and digestion}

Sixteen fecal samples were subjected to an established protocol for protein extraction from stool ${ }^{69}$. Specifically, fecal samples (mean weight $102 \mathrm{mg}$ ) were resuspended in extraction buffer ( $2 \%$ sodium dodecyl sulfate, $100 \mathrm{mM}$ DTT, $20 \mathrm{mM}$ Tris-HCl pH 8.8), adding $250 \mu \mathrm{l}$ of buffer per $100 \mathrm{mg}$ of feces. Samples were incubated at $95^{\circ} \mathrm{C}$ for $20 \mathrm{~min}$ in agitation (500 rpm) in a Thermomixer Comfort (Eppendorf, Hamburg, Germany) and then subjected to bead beating as follows, after adding a steel bead $(5 \mathrm{~mm}$ diameter; Qiagen) to each sample. Sequentially, samples were: incubated at $-80^{\circ} \mathrm{C}$ for $10 \mathrm{~min}$; subjected to bead beating for $10 \mathrm{~min}(30 \mathrm{cycles} / \mathrm{s}$ in a TissueLyser LT mechanical homogenizer, Qiagen); incubated at $-80^{\circ} \mathrm{C}$ for $10 \mathrm{~min}$; incubated at $95^{\circ} \mathrm{C}$ for $10 \mathrm{~min}$; subjected to bead beating for $10 \mathrm{~min}$ (30 cycles/s); centrifuged at $20,000 \times g$ for $10 \mathrm{~min}$ at $4^{\circ} \mathrm{C}$. The final supernatant was collected as the fecal protein extract.

Protein extracts were processed according to a modified FASP protocol $^{70-72}$. Twenty microlitres of each protein extract were diluted to $400 \mu \mathrm{l}$ with UA solution (8 M urea in $100 \mathrm{mM}$ Tris- $\mathrm{HCl}, \mathrm{pH}$ 8.8), filtered using an Ultrafree-MC centrifugal filter $(0.22 \mu \mathrm{m}$; Merck Millipore, Billerica, MA, USA), according to manufacturers' instructions, and loaded onto an Amicon Ultra-0.5 (cutoff $10 \mathrm{kDa}$ ) filtration device (Merck Millipore) and centrifuged at $14,000 \times g$ for $15 \mathrm{~min}$. Then, sequentially, each sample was subjected to addition of $200 \mu \mathrm{l}$ of UA solution and centrifugation (as described above); addition of $100 \mu \mathrm{l}$ of $50 \mathrm{mM}$ iodoacetamide in UA solution, incubation at RT for $20 \mathrm{~min}$, and centrifugation; addition of $100 \mu \mathrm{l}$ of UA solution and centrifugation (twice); addition of $100 \mu \mathrm{l}$ of $50 \mathrm{mM}$ ammonium bicarbonate and centrifugation (twice); addition of $100 \mu \mathrm{l}$ of trypsin solution ( $1 \mu \mathrm{g}$ in $50 \mathrm{mM}$ ammonium bicarbonate) and incubation at $37^{\circ} \mathrm{C}$ overnight. Peptide mixtures were collected by centrifugation, followed by an additional elution with $100 \mu$ l of a $20 \%$ acetonitrile, $0.2 \%$ formic acid solution. Finally, peptide mixtures were brought to dryness and reconstituted in $0.2 \%$ formic acid. Peptide mixtures concentration was estimated by measuring absorbance at $280 \mathrm{~nm}$ with a NanoDrop 2000 spectrophotometer (Thermo Fisher Scientific, Waltham, MS, USA), using dilutions of the MassPREP E. coli Digest Standard (Waters, Milford, MA, USA) to generate a calibration curve.

\section{Liquid chromatography-mass spectrometry analysis}

Liquid chromatography (LC)-tandem mass spectrometry (MS/MS) analyses were performed on an LTQ Orbitrap Velos mass spectrometer (Thermo Fisher Scientific), operating with an EASY-spray source, interfaced with an UltiMate 3000 RSLCnano LC system (Thermo Fisher Scientific). Each sample was run once and analyses were run in a randomized order.

Peptide mixtures ( $4 \mu \mathrm{g}$ per run) were loaded, concentrated, and desalted on a trapping pre-column (Acclaim PepMap C18, $75 \mu \mathrm{m} \times 2 \mathrm{~cm}$ nanoViper, $3 \mu \mathrm{m}, 100 \AA$, Thermo Fisher Scientific), using $0.2 \%$ formic acid at a flow rate of $5 \mu \mathrm{l} / \mathrm{min}$. The peptide separation was performed with a C18 EASY-spray column (PepMap RSLC C18, $75 \mu \mathrm{m} \times 50 \mathrm{~cm}, 2 \mu \mathrm{m}, 100 \AA$, Thermo Fisher Scientific) at $35^{\circ} \mathrm{C}$ with a flow rate of $250 \mathrm{~nL} / \mathrm{min}$ for $247 \mathrm{~min}$, using the following two-step gradient of eluent B $(0.2 \%$ formic acid in $95 \%$ ACN) in eluent $A(0.2 \%$ formic acid in $5 \%$ ACN): $2.5-37.5 \%$ for $242 \mathrm{~min}$ and $37.5-99 \%$ for $5 \mathrm{~min}$. 
The mass spectrometer was set up in a data-dependent MS/MS mode, where a full scan spectrum (from 375 to $2000 \mathrm{~m} / \mathrm{z}$ ) is followed by MS/MS spectra, under the direct control of the Xcalibur software (v.2.2 SP1). The instrument operated in positive mode. The temperature of the ion transfer capillary and the spray voltage were set to $250^{\circ} \mathrm{C}$ and $1.85 \mathrm{kV}$, respectively. Full scans and MS/MS spectra were acquired in the Orbitrap with resolutions of 60,000 and 7500 at $400 \mathrm{~m} / \mathrm{z}$, respectively. The automatic gain control was set to $1,000,000$ ions, and the lock mass option was enabled on a protonated polydimethylcyclosiloxane background ion as an internal recalibration for accurate mass measurements. Peptide ions were selected as the 10 most intense peaks of the previous scan; the signal threshold for triggering an MS/MS event was set to 500 counts, and dynamic exclusion was set to $30 \mathrm{~s}$. Higher-energy collisional dissociation was used as the fragmentation method, by applying a $35 \%$ value for normalized collision energy, an isolation width of $\mathrm{m} / \mathrm{z} 3.0$, a $Q$-value of 0.25 , and an activation time of $0.1 \mathrm{~ms}$. Nitrogen was used as the collision gas.

\section{Peptide identification and annotation}

Peptide identification was carried out using the Proteome Discoverer informatics platform (v.2.4.1.15; Thermo Fisher Scientific), with Sequest-HT as a search engine and Percolator for peptide validation (FDR $<1 \%$ ). Search parameters were set as follows: precursor mass threshold 350-5000 Da; minimum peak count 6; signal-to-noise threshold 2; enzyme trypsin; maximum missed cleavage sites 2; peptide length range 5-50 amino acids; precursor mass tolerance $10 \mathrm{ppm}$; fragment mass tolerance $0.02 \mathrm{Da}$; dynamic modification methionine oxidation; static modification cysteine carbamidomethylation.

Quantification was carried out using the Proteome Discoverer nodes "Minora Feature Detector", "Feature Mapper", and "Precursor lons Quantifier". The integrated peak area of the most abundant peak at the apex of the chromatographic profile was used as a quantitative measure, after being subjected to a normalization step based on the total peptide intensity of the samples, based on the results of a recent comparison among MS1-based label-free protein quantification tools $\mathrm{s}^{73}$.

The identification node was built on a combination of databases. Two microbial sequence databases were used: (i) a collection of metagenomic sequences obtained in house from rat fecal samples $(13,163,507$ sequences; file name: DB1.fasta); (ii) a publicly available rat metagenomic dataset (ftp://ftp.cngb.org/pub/SciRAID/Microbiome/rat/GeneCatalog/rat_geneset. pep.gz; 5,130,167 sequences; file name: DB2.fasta) $)^{74}$. Moreover, two additional databases, containing host (reference proteome for Rattus norvegicus; https://www.uniprot.org/proteomes/UP000002494 release 2021_01; 29,936 sequences; file name: DB3.fasta) and food (reference proteome for Glycine max; https://www.uniprot.org/proteomes/UP000008827 release 2020_12; 74,863 sequences; file name: DB4.fasta) protein sequences, were employed.

The file named PeptideGroups.txt (available in the ProteomeXchange repository, see "Data availability statement" for details) was used as input for statistical analyses.

Taxonomic annotation was obtained according to the following steps: first, protein sequences were subjected to a DIAMOND (v.0.8.22) search against the NCBI-nr database (2021/05 update), using the blastp command with default parameters ${ }^{75}$; then, DIAMOND outputs were loaded on MEGAN (v.6.19.9) ${ }^{76}$ using default parameters. Only species annotated with binomial nomenclature were considered for differential analysis; the species for which a binomial nomenclature was not available are marked in red in Supplementary Data 3.

Functional characterization allowed the classification of protein sequences according to UniProt protein families and metabolic pathways $^{77}$, COG of proteins ${ }^{78}$, KOG $^{79}$, and CAZy ${ }^{80}$.

Protein families and metabolic pathways were obtained through the following steps: first, the identified protein sequences were aligned against a database containing all bacterial sequences from UniProtKB/Swiss-Prot (release 2021_05) using DIAMOND (blastp module, e-value threshold $10^{-5}$ ); then, UniProtKB/Swiss-Prot accession numbers were exploited to retrieve specific information from the UniProt website (https://www.uniprot.org) via the "retrieve" tool. COG, KOG, and CAZy information was achieved using the eggnog-mapper package $(v \cdot 2.0 .1)^{81}$ available in a Galaxy server (https://proteomics.usegalaxy.eu) ${ }^{82}$, according to the following parameters: eggnog database v.2.0; min e-value threshold 0.001 ; $\mathrm{min}$ bit score threshold 60 . In the case of the assignment of a single peptide sequence to multiple protein entries, the first protein entry in the list having a valid blastp output was selected.

\section{Statistical analysis and graph generation}

PCoA plots were generated using the MicrobiomeAnalyst web application (https://www.microbiomeanalyst.ca), which also calculates the statistical significance of group clustering through a PERMANOVA test ${ }^{83}$.

Differential analysis of $16 \mathrm{~S}$ rRNA gene sequencing data was performed on count data at various taxonomic levels, obtained by aggregating ASVs based on their taxonomy assignment, through the MicrobiomeAnalyst web application. Features with valid values in less than six samples in at least one of the compared groups were filtered out. Count data were transformed prior to statistical testing according to the Relative Log Expression method ${ }^{84}$. Differential abundance analysis was then carried out using the edgeR algorithm ${ }^{85}$. The $p$-value correction for multiple tests was performed by calculating an $\mathrm{FDR}^{86}$, and results were considered as significant for FDR $<0.1$.

Differential analysis of metaproteomic data was performed using the Perseus computational platform (v.1.6.15.0) ${ }^{87}$, using as inputs peptide area values (aggregated based on the functional and taxonomic annotation levels), according to the following steps: (i) data log-transformation: abundance data were subjected to binary logarithmic transformation to approximate a normal distribution, subsequently verified using the Shapiro-Wilk test; (ii) protein filtering: features not reaching $75 \%$ valid values in at least one group were filtered out; (iii) missing value (MV) replacement: MVs were replaced with a constant value, calculated for each comparison as the binary logarithm of the minimum of the distribution (approximated to the nearest integer) minus 1; (iv) differential analysis: differential protein abundances between groups were tested with a two-tail Student's $t$ test; $(v)$ correction for multiple testing: FDR was calculated based on permutation-adjustment considering $q=0.1$ as the threshold of significance. An abundance log-ratio (LR) was also computed using Microsoft Excel (Redmond, WA, USA) as a quantitative measure of the change in abundance of a feature between the two sample groups. The LR was calculated for each feature, starting from its original abundance data, as the binary logarithm of the ratio between the mean abundances measured in the two sample groups (after summing a background correction factor equal to 1000 to the mean abundances) for that feature.

Heatmaps were generated using the web application Morpheus (https:// software.broadinstitute.org/morpheus/). The galactose metabolism pathway was reconstructed based on the corresponding KEGG pathway map, available at http://www.genome.jp/kegg/pathway.html $\mathrm{l}^{79}$, and further refined based on recently reviewed data concerning galactose metabolism in lactic bacteria ${ }^{88}$.

\section{Reporting summary}

Further information on research design is available in the Nature Research Reporting Summary linked to this article.

\section{DATA AVAILABILITY}

The mass spectrometry proteomics data (including all sequence databases) have been deposited to the ProteomeXchange Consortium via the PRIDE ${ }^{89}$ partner repository with the dataset identifier PXD024509. The 16S rRNA gene sequencing data have been deposited to the European Nucleotide Archive (ENA) with the identifier PRJEB46472.

Received: 10 March 2021; Accepted: 3 November 2021; Published online: 03 December 2021

\section{REFERENCES}

1. Weindruch, R. \& Sohal, R. S. Seminars in medicine of the Beth Israel Deaconess Medical Center. Caloric intake and aging. N. Engl. J. Med. 337, 986-994 (1997).

2. Most, J., Tosti, V., Redman, L. M. \& Fontana, L. Calorie restriction in humans: an update. Ageing Res. Rev. 39, 36-45 (2017).

3. Nencioni, A., Caffa, I., Cortellino, S. \& Longo, V. D. Fasting and cancer: molecular mechanisms and clinical application. Nat. Rev. Cancer 18, 707-719 (2018).

4. Mattson, M. P., Longo, V. D. \& Harvie, M. Impact of intermittent fasting on health and disease processes. Ageing Res. Rev. 39, 46-58 (2017).

5. de Cabo, R. \& Mattson, M. P. Effects of intermittent fasting on health, aging, and disease. N. Engl. J. Med. 381, 2541-2551 (2019).

6. Chaix, A., Zarrinpar, A., Miu, P. \& Panda, S. Time-restricted feeding is a preventative and therapeutic intervention against diverse nutritional challenges. Cell Metab. 20, 991-1005 (2014). 
7. Tinsley, G. M. et al. Time-restricted feeding in young men performing resistance training: a randomized controlled trial. Eur. J. Sport Sci. 17, 200-207 (2017).

8. Moro, T. et al. Effects of eight weeks of time-restricted feeding (16/8) on basal metabolism, maximal strength, body composition, inflammation, and cardiovascular risk factors in resistance-trained males. J. Transl. Med. 14, 290 (2016).

9. LeCheminant, J. D., Christenson, E., Bailey, B. W. \& Tucker, L. A. Restricting nighttime eating reduces daily energy intake in healthy young men: a short-term cross-over study. Br. J. Nutr. 110, 2108-2113 (2013).

10. Panda, S. Circadian physiology of metabolism. Science 354, 1008-1015 (2016).

11. Longo, V. D. \& Panda, S. Fasting, circadian rhythms, and time-restricted feeding in healthy lifespan. Cell Metab. 23, 1048-1059 (2016).

12. Serra, M., Marongiu, F., Pisu, M. G., Serra, M. \& Laconi, E. Time-restricted feeding delays the emergence of the age-associated, neoplastic-prone tissue landscape. Aging 11, 3851-3863 (2019).

13. Ismail, S., Manaf, R. A. \& Mahmud, A. Comparison of time-restricted feeding and Islamic fasting: a scoping review. East Mediterr. Health J. 25, 239-245 (2019).

14. Manoogian, E. N. C. \& Panda, S. Circadian rhythms, time-restricted feeding, and healthy aging. Ageing Res. Rev. 39, 59-67 (2017).

15. Cangemi, A. et al. Dietary restriction: could it be considered as speed bump on tumor progression road? Tumour Biol. 37, 7109-7118 (2016).

16. Duncan, M. J. et al. Restricting feeding to the active phase in middle-aged mice attenuates adverse metabolic effects of a high-fat diet. Physiol. Behav. 167, 1-9 (2016).

17. Hatori, M. et al. Time-restricted feeding without reducing caloric intake prevents metabolic diseases in mice fed a high-fat diet. Cell Metab. 15, 848-860 (2012).

18. Mitchell, S. J. et al. Daily fasting improves health and survival in male mice independent of diet composition and calories. Cell Metab. 29, 221-228.e3 (2019).

19. Illiano, P., Brambilla, R. \& Parolini, C. The mutual interplay of gut microbiota, diet and human disease. FEBS J. 287, 833-855 (2020).

20. David, L. A. et al. Diet rapidly and reproducibly alters the human gut microbiome. Nature 505, 559-563 (2014).

21. Ren, J. et al. Alteration in gut microbiota caused by time-restricted feeding alleviate hepatic ischaemia reperfusion injury in mice. J. Cell. Mol. Med. 23, 1714-1722 (2019).

22. Zeb, F. et al. Time-restricted feeding is associated with changes in human gut microbiota related to nutrient intake. Nutrition 78, 1-11 (2020).

23. Hu, D. et al. Time-restricted feeding causes irreversible metabolic disorders and gut microbiota shift in pediatric mice. Pediatr. Res. 85, 518-526 (2019).

24. Hu, D., Ye, Y., Mao, Y., Liao, W. \& Xu, W. Time-restricted feeding during childhood has persistent effects on mice commensal microbiota. Ann. Transl. Med. 7, 556-556 (2019).

25. Heintz-Buschart, A. \& Wilmes, P. Human gut microbiome: function matters. Trends Microbiol. 26, 563-574 (2018)

26. Mittelstadt, S. W., Hemenway, C. L. \& Spruell, R. D. Effects of fasting on evaluation of gastrointestinal transit with charcoal meal. J. Pharmacol. Toxicol. Methods 52, 154-158 (2005).

27. Turroni, F. et al. Molecular dialogue between the human gut microbiota and the host: a Lactobacillus and Bifidobacterium perspective. Cell. Mol. Life Sci. 71, 183-203 (2014)

28. Li, D. et al. Microbial biogeography and core microbiota of the rat digestive tract. Sci. Rep. 7, 45840 (2017).

29. Nava, G. M., Carbonero, F., Croix, J. A., Greenberg, E. \& Gaskins, H. R. Abundance and diversity of mucosa-associated hydrogenotrophic microbes in the healthy human colon. ISME J. 6, 57-70 (2012)

30. $\mathrm{Wu}, \mathrm{M}$. et al. The differences between luminal microbiota and mucosal microbiota in mice. J. Microbiol. Biotechnol. 30, 287-295 (2020).

31. Derrien, M., Vaughan, E. E., Plugge, C. M. \& de Vos, W. M. Akkermansia muciniphila gen. nov., sp. nov., a human intestinal mucin-degrading bacterium. Int. J. Syst. Evol. Microbiol. 54, 1469-1476 (2004).

32. van Passel, M. W. J. et al. The genome of Akkermansia muciniphila, a dedicated intestinal mucin degrader, and its use in exploring intestinal metagenomes. PLoS ONE 6, 1-8 (2011).

33. Kostopoulos, I. et al. A continuous battle for host-derived glycans between a mucus specialist and a glycan generalist in vitro and in vivo. Front. Microbiol. 12, 1518 (2021).

34. Belzer, C. et al. Microbial metabolic networks at the mucus layer lead to dietindependent butyrate and vitamin $B_{12}$ production by intestinal symbionts. $m B i o$ 8, e00770-17 (2017).

35. Yang, M. et al. Beneficial effects of newly isolated Akkermansia muciniphila strains from the human gut on obesity and metabolic dysregulation. Microorganisms $\mathbf{8}$, 1413 (2020).

36. de Vos, W. M. Microbe profile: Akkermansia muciniphila: a conserved intestinal symbiont that acts as the gatekeeper of our mucosa. Microbiology 163, 646-648 (2017)
37. Everard, A. et al. Cross-talk between Akkermansia muciniphila and intestinal epithelium controls diet-induced obesity. Proc. Nati. Acad. Sci. USA 110, 9066-9071 (2013)

38. Maifeld, A. et al. Fasting alters the gut microbiome reducing blood pressure and body weight in metabolic syndrome patients. Nat. Commun. 12, 1970 (2021).

39. Schneeberger, M. et al. Akkermansia muciniphila inversely correlates with the onset of inflammation, altered adipose tissue metabolism and metabolic disorders during obesity in mice. Sci. Rep. 5, 16643 (2015).

40. Shin, J. et al. Elucidation of Akkermansia muciniphila probiotic traits driven by mucin depletion. Front. Microbiol. 10, 1137 (2019).

41. Oberto, J., Nabti, S., Jooste, V., Mignot, H. \& Rouviere-Yaniv, J. The HU regulon is composed of genes responding to anaerobiosis, acid stress, high osmolarity and SOS induction. PLOS ONE 4, 1-20 (2009).

42. Kamashev, D. et al. Comparison of histone-like HU protein DNA-binding properties and HU/IHF protein sequence alignment. PLOS ONE 12, 1-24 (2017).

43. Kar, S. \& Adhya, S. Recruitment of HU by piggyback: a special role of GalR in repressosome assembly. Genes Dev. 15, 2273-2281 (2001).

44. Karcher, N. et al. Genomic diversity and ecology of human-associated Akkermansia species in the gut microbiome revealed by extensive metagenomic assembly. Genome Biol. 22, 209 (2021).

45. Kleiner, M. et al. Assessing species biomass contributions in microbial communities via metaproteomics. Nat. Commun. 8, 1558 (2017).

46. Jizhong, Z. et al. High-throughput metagenomic technologies for complex microbial community analysis: open and closed formats. mBio 6, e02288-14 (2021).

47. Tanca, A. et al. Metaproteogenomics reveals taxonomic and functional changes between cecal and fecal microbiota in mouse. Front. Microbiol. 8, 391 (2017).

48. Badet, C. \& Thebaud, N. B. Ecology of lactobacilli in the oral cavity: a review of literature. Open Microbiol. J. 2, 38-48 (2008).

49. Nishiyama, K., Sugiyama, M. \& Mukai, T. Adhesion properties of lactic acid bacteria on intestinal mucin. Microorganisms 4, 34 (2016).

50. Wu, M. et al. The dynamic changes of gut microbiota in Muc2 deficient mice. Int J. Mol. Sci. 19, 2809 (2018).

51. Zúñiga, M., Monedero, V. \& Yebra, M. J. Utilization of host-derived glycans by intestinal Lactobacillus and Bifidobacterium species. Front. Microbiol. 9, 1917 (2018).

52. Dao, M. C. et al. Akkermansia muciniphila and improved metabolic health during a dietary intervention in obesity: relationship with gut microbiome richness and ecology. Gut 65, 426-436 (2016).

53. Desai, M. S. et al. A dietary fiber-deprived gut microbiota degrades the colonic mucus barrier and enhances pathogen susceptibility. Cell 167, 1339-1353.e21 (2016).

54. Bell, A. \& Juge, N. Mucosal glycan degradation of the host by the gut microbiota. Glycobiology 31, 691-696 (2020).

55. Sekar, K. et al. Synthesis and degradation of FtsZ quantitatively predict the first cell division in starved bacteria. Mol. Syst. Biol. 14, e8623 (2018).

56. Rhimi, M. et al. The secreted L-arabinose isomerase displays anti-hyperglycemic effects in mice. Microb. Cell Fact. 14, 1-8 (2015).

57. Cox, L. M. et al. Calorie restriction slows age-related microbiota changes in an Alzheimer's disease model in female mice. Sci. Rep. 9, 1-14 (2019).

58. Zagato, E. et al. Endogenous murine microbiota member Faecalibaculum rodentium and its human homologue protect from intestinal tumour growth. Nat Microbiol. 5, 511-524 (2020).

59. Wang, H., Xia, P., Lu, Z., Su, Y. \& Zhu, W. Metabolome-microbiome responses of growing pigs induced by time-restricted feeding. Front. Vet. Sci. 8, 681202 (2021).

60. Sarmiento, M. R. A. et al. Obesity, xenobiotic intake and antimicrobial-resistance genes in the human gastrointestinal tract: a comparative study of eutrophic overweight and obese individuals. Genes (Basel) 10, 349 (2019).

61. Clemente, J. C. et al. The microbiome of uncontacted Amerindians. Sci. Adv. 1, e1500183 (2015)

62. Martin, M. Cutadapt removes adapter sequences from high-throughput sequencing reads. EMBnet J. 17, 10 (2011).

63. Bolyen, E. et al. Reproducible, interactive, scalable and extensible microbiome data science using QIIME 2. Nat. Biotechnol. 37, 852-857 (2019).

64. Callahan, B. J. et al. DADA2: high-resolution sample inference from Illumina amplicon data. Nat. Methods 13, 581-583 (2016).

65. Quast, C. et al. The SILVA ribosomal RNA gene database project: improved data processing and web-based tools. Nucleic Acids Res. 41, D590-D596 (2013).

66. Bokulich, N. A. et al. Optimizing taxonomic classification of marker-gene amplicon sequences with QIIME 2's q2-feature-classifier plugin. Microbiome 6, 90 (2018).

67. Qiong, W., Garrity, G. M., Tiedje, J. M. \& Cole, J. R. Naïve Bayesian classifier for rapid assignment of rRNA sequences into the new bacterial taxonomy. Appl. Environ. Microbiol. 73, 5261-5267 (2007)

68. McMurdie, P. J. \& Holmes, S. phyloseq: an R package for reproducible interactive analysis and graphics of microbiome census data. PLOS ONE 8, e61217 (2013). 
69. Tanca, A. et al. A straightforward and efficient analytical pipeline for metaproteome characterization. Microbiome 2, 49 (2014).

70. Wiśniewski, J. R. et al. Universal sample preparation method for proteome analysis. Nat. Methods 6, 359-362 (2009).

71. Wiśniewski, J. R. Filter-aided sample preparation for proteome analysis. Methods Mol. Biol. 1841, 3-10 (2018).

72. Tanca, A., Biosa, G., Pagnozzi, D., Addis, M. F. \& Uzzau, S. Comparison of detergent-based sample preparation workflows for LTQ-Orbitrap analysis of the Escherichia coli proteome. Proteomics 13, 2597-2607 (2013).

73. Palomba, A. et al. Comparative evaluation of MaxQuant and proteome discoverer MS1-based protein quantification tools. J. Proteome Res. 20, 3497-3507 (2021).

74. Pan, H. et al. A gene catalogue of the Sprague-Dawley rat gut metagenome. Gigascience 7, giy055 (2018).

75. Buchfink, B., Xie, C. \& Huson, D. H. Fast and sensitive protein alignment using DIAMOND. Nat. Methods 12, 59-60 (2015).

76. Huson, D. H. et al. MEGAN community edition-interactive exploration and analysis of large-scale microbiome sequencing data. PLOS Comput. Biol. 12, e1004957 (2016).

77. UniProt Consortium. UniProt: the universal protein knowledgebase in 2021. Nucleic Acids Res. 49, D480-D489 (2021).

78. Galperin, M. Y. et al. COG database update: focus on microbial diversity, model organisms, and widespread pathogens. Nucleic Acids Res. 49, D274-D281 (2021).

79. Kanehisa, M., Furumichi, M., Sato, Y., Ishiguro-Watanabe, M. \& Tanabe, M. KEGG: integrating viruses and cellular organisms. Nucleic Acids Res. 49, D545-D551 (2021).

80. Lombard, V., Golaconda Ramulu, H., Drula, E., Coutinho, P. M. \& Henrissat, B. The carbohydrate-active enzymes database (CAZy) in 2013. Nucleic Acids Res. 42, D490-D495 (2014).

81. Huerta-Cepas, J. et al. Fast genome-wide functional annotation through orthology assignment by eggNOG-mapper. Mol. Biol. Evol. 34, 2115-2122 (2017).

82. Jagtap, P. D. et al. Metaproteomic analysis using the Galaxy framework. Proteomics 15, 3553-3565 (2015).

83. Dhariwal, A. et al. MicrobiomeAnalyst: a web-based tool for comprehensive statistical, visual and meta-analysis of microbiome data. Nucleic Acids Res. 45, W180-W188 (2017).

84. Anders, S. \& Huber, W. Differential expression analysis for sequence count data. Genome Biol. 11, R106 (2010).

85. Anders, S. et al. Count-based differential expression analysis of RNA sequencing data using R and Bioconductor. Nat. Protoc. 8, 1765-1786 (2013).

86. Benjamini, Y. \& Hochberg, Y. Controlling the false discovery rate: a practical and powerful approach to multiple testing. J. R. Stat. Soc. Ser. B 57, 289-300 (1995).

87. Tyanova, S. et al. The Perseus computational platform for comprehensive analysis of (prote)omics data. Nat. Methods 13, 731-740 (2016).

88. Iskandar, C. F., Cailliez-Grimal, C., Borges, F. \& Revol-Junelles, A.-M. Review of lactose and galactose metabolism in Lactic Acid Bacteria dedicated to expert genomic annotation. Trends Food Sci. Technol. 88, 121-132 (2019).

89. Deutsch, E. W. et al. The ProteomeXchange consortium in 2020: enabling 'big data' approaches in proteomics. Nucleic Acids Res. 48, D1145-D1152 (2020).

\section{ACKNOWLEDGEMENTS}

This work was supported by Sardegna Ricerche - Science and Technology Park of Sardinia (grant program art.9 LR 20/2015 to Porto Conte Ricerche) and by the University of Sassari (grants FAR 2019/2020 to S.U. and A.T.).

\section{AUTHOR CONTRIBUTIONS}

A.P., A.T., E.L., and S.U. designed the study. M.S., F.M., and E.L. performed animal experiments. A.P. performed metaproteomic analysis. D.P. contributed to the metaproteomic analysis. CF performed 16S rRNA gene sequencing analysis. A.P., A.T., M.A., and R.S. performed data analysis. A.P., A.T., and S.U. wrote the paper. M.A., R.S., M.S., F.M., C.F., D.P., and E.L. contributed to critically revising the paper. A.P. and A.T. contributed equally to this work. All the authors have read and approved the final version of the paper.

\section{COMPETING INTERESTS}

The authors declare no competing interests.

\section{ADDITIONAL INFORMATION}

Supplementary information The online version contains supplementary material available at https://doi.org/10.1038/s41522-021-00256-x.

Correspondence and requests for materials should be addressed to Sergio Uzzau.

Reprints and permission information is available at http://www.nature.com/ reprints

Publisher's note Springer Nature remains neutral with regard to jurisdictional claims in published maps and institutional affiliations.

(C) Open Access This article is licensed under a Creative Commons Attribution 4.0 International License, which permits use, sharing, adaptation, distribution and reproduction in any medium or format, as long as you give appropriate credit to the original author(s) and the source, provide a link to the Creative Commons license, and indicate if changes were made. The images or other third party material in this article are included in the article's Creative Commons license, unless indicated otherwise in a credit line to the material. If material is not included in the article's Creative Commons license and your intended use is not permitted by statutory regulation or exceeds the permitted use, you will need to obtain permission directly from the copyright holder. To view a copy of this license, visit http://creativecommons. org/licenses/by/4.0/.

(c) The Author(s) 2021 